\title{
Characteristic Curve Fitting of Capacitive Rainfall Sensor Based on BP Neural Network \\ Long Zhang ${ }^{a}$, Song Ye ${ }^{b,{ }^{*}}$, Shu-dao Zhou ${ }^{c}$ and Feng Liu ${ }^{d}$
}

\author{
College of Meteorology and Oceanography, PLA University of Science and Technology, Nanjing, \\ 211101, China \\ aemail:zhanglonglxy@163.com, bemail:yesong999@hotmail.com*, 'email:zhousd70131@sina.com \\ , demail:li_ufeng@126.com, *corresponding author
}

Keywords: Capacitive Rainfall Sensor, Curve Fitting, Least Square Method, BP Neural Network, Mean Square Error

\begin{abstract}
Rainfall is a kind of common weather phenomenon. Accurate measurement of rainfall is of great significance for weather and natural disasters forecasting. In this paper, the capacitive sensor was applied to the measurement of rainfall and the working principle was introduced. Furthermore, the output characteristic curve of the sensor was fitted by BP neural network. The sensor's calibration data were taken as training samples and BP network model was established. The results showed that the fitting algorithm based on BP neural network had faster convergence speed and higher accuracy, and its fitting error was much smaller than that of the least square method.
\end{abstract}

\section{Introduction}

Rainfall is a kind of common weather phenomenon, which has an important influence on environment, agriculture, military action and so on. High intensity rainfall often leads to severe casualty and property losses. Therefore, the rainfall measurement has become a research focus in many fields, such as meteorology, hydrology and environment.

Since 1970s, there have been many kinds of rainfall measuring instruments based on the optical method, vibration method and other measuring principles[1]. Due to the technical level, the development of rainfall measuring instruments is backward in domestic. The rainfall measuring instruments used in the meteorological observation has a large measurement error.

The capacitive sensor is applied to the rainfall measurement in this paper. Due to the influence of edge effect and parasitic capacitance, the sensor's output characteristic curve has large nonlinear error. The traditional linear fitting method cannot describe the input-output characteristics of the sensor accurately. In order to reduce the nonlinear error, a variety of methods are used to compensate the nonlinear characteristics of sensors, such as least square method, cubic spline function method and nonlinear inverse function method [2-4]. However, the compensation error of cubic spline function method and nonlinear inverse function method is large; the least square method is limited by the amount of fitting data, and it is difficult to obtain polynomial coefficients when the amount of fitting data is large. BP neural network has the characteristics of simple structure and fast convergence speed, which can approximate the nonlinear function with any precision. Therefore, it is suitable for the fitting of sensor's characteristic curve [5].In this paper, the least square method and BP neural network were used to fit the characteristic curve of the capacitive rain sensor, and the fitting results were compared. The results showed that the BP neural network had stronger nonlinear mapping ability, and its fitting accuracy was better than that of the least square method.

\section{The Working Principle Of Capacitive Rainfall Sensor}

Sensor's structure. The sensor discussed in this paper is coaxial cylindrical structure, as shown in Fig 1. 


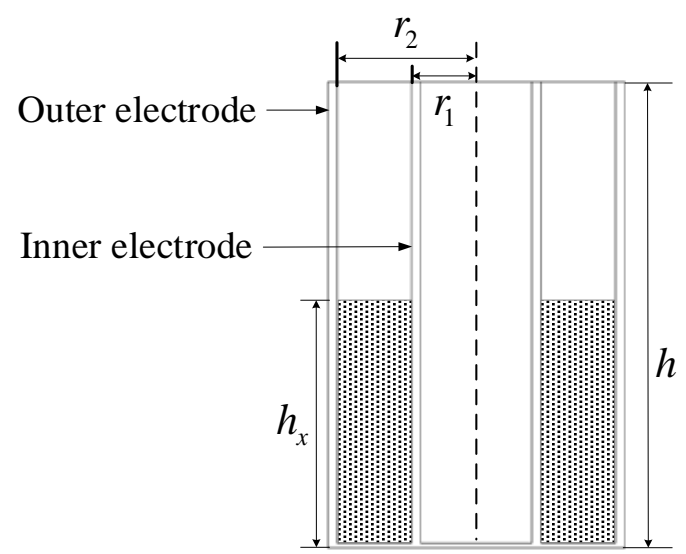

Fig 1 Structural diagram of the sensor

According to the principle of variable dielectric constant capacitance sensor, the sensor's capacitance value $C$ can be calculated by Eq1, where $r_{1}$ and $r_{2}$ are the outer radius of inner electrode and the inner radius of outer electrode, respectively; $\varepsilon$ and $\varepsilon_{0}$ are the dielectric constant of rainwater and air, respectively; $h$ and $h_{x}$ are the height of electrode and rainwater, respectively.

$$
C=\frac{2 \pi \varepsilon_{0} h}{\ln \left(r_{2} / r_{1}\right)}+\frac{2 \pi\left(\varepsilon-\varepsilon_{0}\right) h_{x}}{\ln \left(r_{2} / r_{1}\right)}
$$

Signal Conditioning Circuit. The double T type diode circuit is selected for the sensor's signal conditioning, as shown in Fig 2. In this circuit, the power supply is a square wave with an amplitude of $\pm U_{\mathrm{E}}$, a duty cycle of $50 \%$, and a period of $T$.

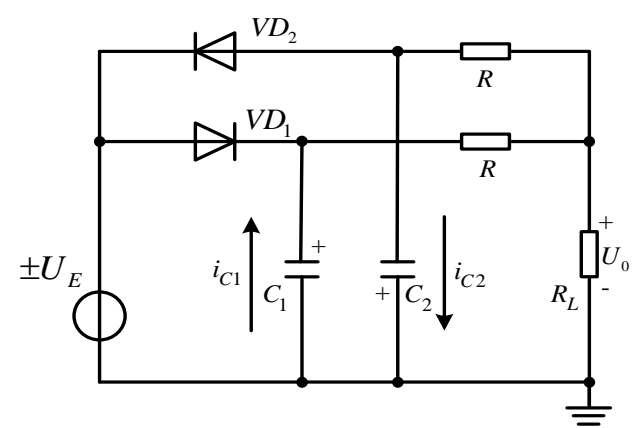

Fig 2 The double T type diode circuit

When the amplitude of power supply is positive, diode $V D_{2}$ is in open circuit condition and diode $V D_{1}$ is in short circuit condition. The initial voltage value of the capacitor $C_{2}$ is $U_{\mathrm{E}}$.According to the time domain analysis method of first order circuit, the average current flowing through the capacitor $C_{2}$ can be calculated by Eq 2 .

$$
I_{C 2}=\frac{1}{T} \frac{R+2 R_{L}}{R+R_{L}} U_{E} C_{2}
$$

In the same way, when the amplitude of power supply is negative, the average current flowing through the capacitor $C_{1}$ can be calculated by Eq 3 .

$$
I_{C 1}=\frac{1}{T} \frac{R+2 R_{L}}{R+R_{L}} U_{E} C_{1}
$$

The voltage on the resistance $R$ can be calculated by Eq 4 .

$$
U_{0}=\frac{R R_{L}\left(R+2 R_{L}\right)}{\left(R+R_{L}\right)^{2}} \frac{U_{E}}{T}\left(C_{1}-C_{2}\right)
$$

Assuming the sensor's capacitance is $C_{1}, C_{2}$ is a fixed value capacitor which has the same initial value as $C_{1}$. By Eq 1 and 4,the relationship between the output voltage of the signal conditioning circuit and the rainfall height can be expressed by Eq 5, where $K$ is a constant related to the 
parameters of the sensor and signal conditioning circuit.

$$
U_{0}=2 \pi K \frac{\left(\varepsilon-\varepsilon_{0}\right)}{\ln \left(r_{2}-r_{1}\right)} h_{x}+K\left(\frac{2 \pi \varepsilon_{0} h}{\ln \left(r_{2}-r_{1}\right)}-C_{2}\right)
$$

By Eq 5, the output voltage $U_{0}$ meets the linear relationship with rainfall height $h_{x}$. In practice, the output characteristic of the sensor is difficult to meet the linear relationship because of the influence of the parasitic capacitance and the edge effect.

\section{BP Neural Network}

The Structure Of BP Neural Network. BP (Back Propagation) neural network was proposed in 1986 by the PDP (Parallel Distributed Procession) group. The typical BP network has 3 layers, including the input layer, the hide layer and the output layer. Its structure is shown in Fig 3.The neurons in two adjacent layers are all connected, and the neurons in the same layer are not connected [6].

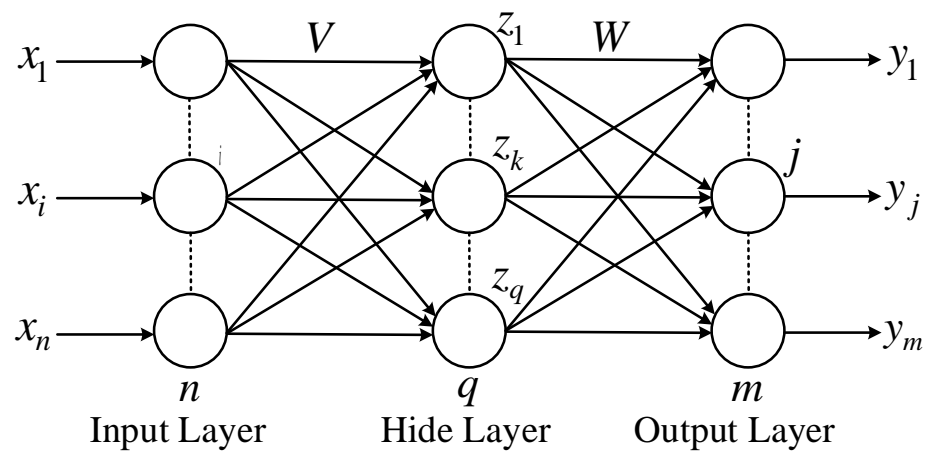

Fig 3 The architecture of BP neural network

The Learning Algorithm Of BP Network. The error back propagation learning algorithm is applied to the BP network. According to the training samples, the connection weights between neurons are adjusted. During the training process, the data is transmitted to next layer from the input layer. On the contrary, the network weights are modified from the output layer along the direction of error reduction[7].

Assume that the input sample is $X_{\mathrm{p}}$, the target sample is $Y_{\mathrm{p}}$ and the network output is $T_{\mathrm{p}}$, the error between the output value and the target value can be reduced gradually by the error back propagation algorithm[8].In the process of information forward propagation, the network output $T_{\mathrm{p}}$ can be calculated. The operation of the network is shown in Eq 6.

$$
T_{P}=F_{n}\left(\ldots\left(F_{2}\left(F_{1}\left(X_{P} W^{(1)}\right) W^{(2)}\right) \cdots\right) W^{(n)}\right)
$$

In the process of error back propagation, the error between the network output $T_{\mathrm{p}}$ and the target sample $Y_{\mathrm{p}}$ can be calculated by Eq 7.

$$
E_{p}=\frac{1}{2} \sum_{j=1}^{m}\left(y_{p j}-o_{p j}\right)^{2}
$$

\section{Model Establishment And Result Analysis}

Least Square Method. The static calibration is carried out for three cycles, and the average value of the output voltage at each calibration point can be calculated. The least square method is used to fit the calibration data, the first order linear fitting results are shown in Fig 4. 


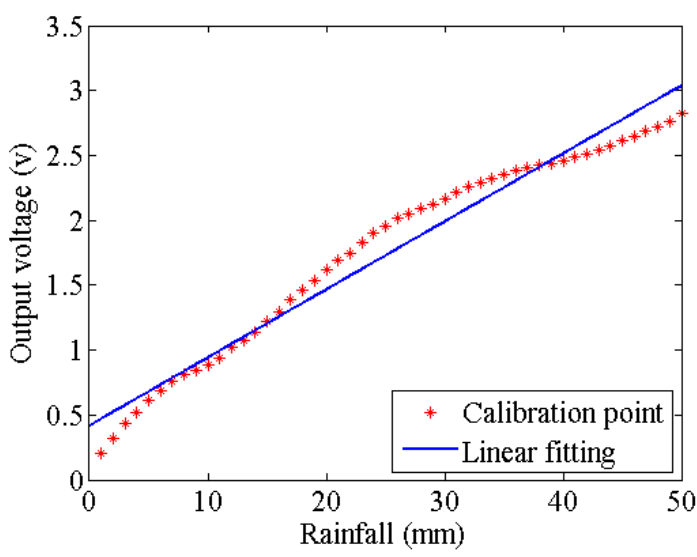

(a) First order fitting curve

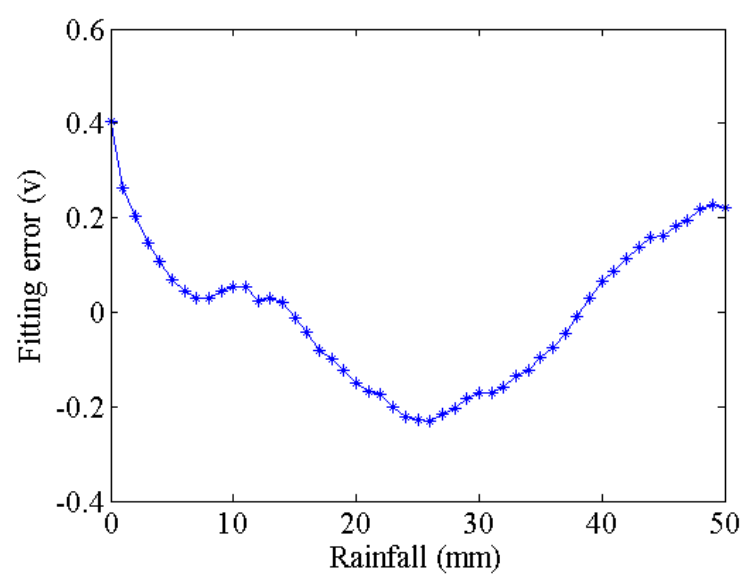

(b) Fitting error curve

Fig 4 The results of first order linear fitting

Due to the influence of parasitic capacitance and edge effect, the nonlinear error of the sensor is large. It is difficult to express the input and output characteristics of the sensor accurately by the first order linear fitting method. In order to improve the fitting precision, the three order polynomial is used to fit the calibration data, and the results are shown in Fig 5.Fig 4 and 5 show that the higher the polynomial order, the smaller the fitting error.

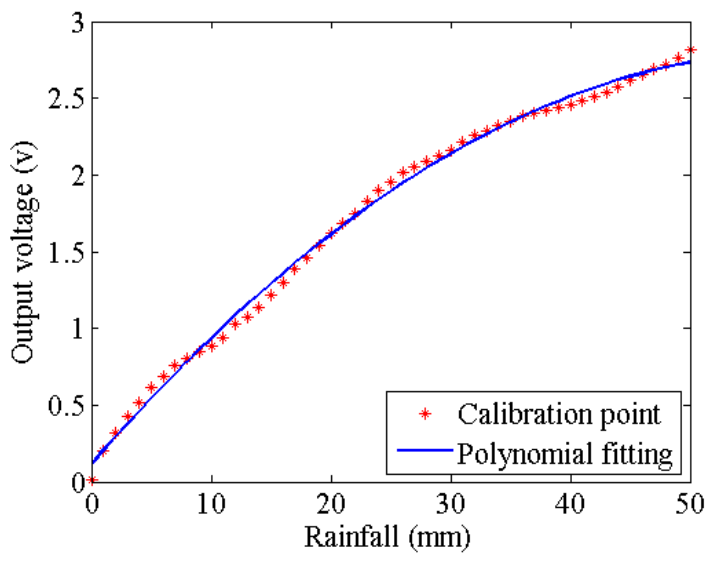

(a) Three order polynomial fitting curve

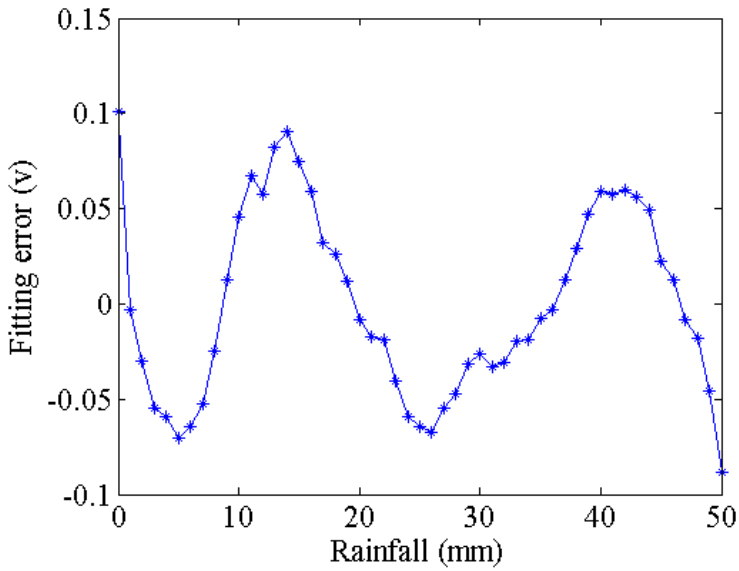

(b) Fitting error curve

Fig 5 The results of three order polynomial fitting

BP Neural Network. The calibration points are used as the network input sample, and the corresponding output voltages are taken as the network target sample. Build up and train the network model using MATLAB. After 36 times of weight and threshold adjustment, the fitting error reaches the required range. The fitting results are shown in Fig 6.

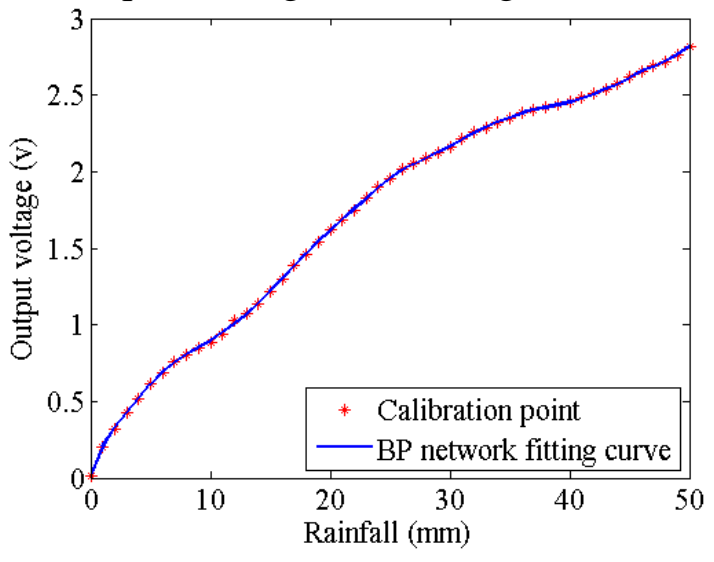

(a)Fitting curve of BP neural network

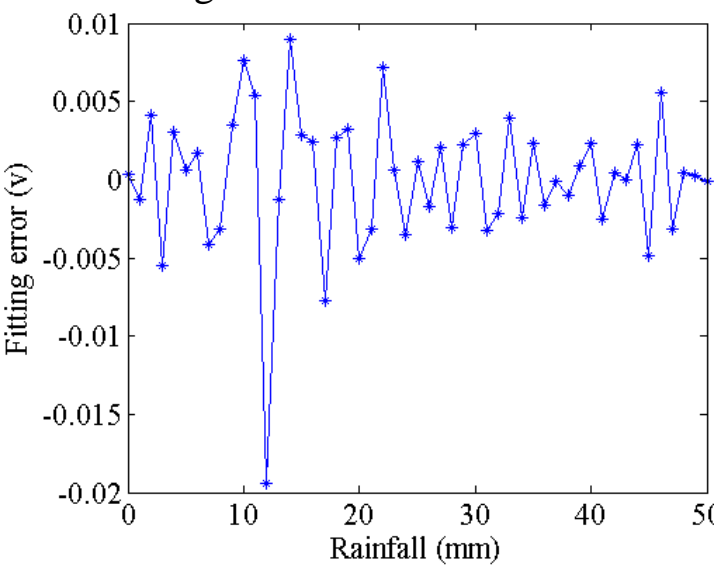

(b) Fitting error curve

Fig 6 The fitting results of BP neural network

Comparison Of Fitting Results. The mean square error is defined as the error comparison criterion, which can be calculated by Eq $8 . N$ is the number of calibration points, and $e_{i}$ is the fitting 
error of each calibration point.

$$
m s e=\frac{1}{N} \sum_{i=1}^{N}\left(e_{i}\right)^{2}
$$

The mean square error and the maximum fitting error of the above three models are calculated respectively, and the results are shown in Table 1.The results show that the fitting effect of BP neural network is obviously better than that of the least square method.

Table 1 Fitting error comparison of three models

\begin{tabular}{cccc}
\hline & $\begin{array}{c}\text { First order fitting } \\
\text { model }\end{array}$ & $\begin{array}{c}\text { Three order fitting } \\
\text { model }\end{array}$ & $\begin{array}{c}\text { BP network } \\
\text { model }\end{array}$ \\
\hline Mean square error & 0.0236 & 0.0024 & $1.960 \times 10^{-5}$ \\
Maximum fitting error & 0.4050 & 0.1011 & 0.0091 \\
\hline
\end{tabular}

The 6 groups of data generated in the calibration process are used to verify the three fitting models. The mean square error and the maximum fitting error of the above three models are calculated respectively, and the results are shown in Table 2.The table show that the fitting effect of BP neural network is obviously better than that of the least square method.

Table 2 Fitting error comparison of six groups of test samples

\begin{tabular}{cccccccc}
\hline & & \multicolumn{5}{c}{ Sample 1 } & \multicolumn{5}{c}{ Sample 2 Sample3Sample4Sample5 Sample 6 } \\
\hline Maximum & BP network model & 0.0084 & 0.0128 & 0.0120 & 0.0129 & 0.0118 & 0.0119 \\
fitting & Three order fitting model & 0.1127 & 0.0950 & 0.1143 & 0.0911 & 0.1142 & 0.0902 \\
error & First order fitting model & 0.4220 & 0.3874 & 0.4254 & 0.3819 & 0.4240 & 0.3890 \\
Mean & BP network model $\left(10^{-5}\right)$ & 1.7289 & 3.4618 & 2.0218 & 2.1698 & 1.7431 & 1.9366 \\
square & Three order fitting model & 0.0024 & 0.0023 & 0.0024 & 0.0023 & 0.0024 & 0.0025 \\
error & First order fitting model & 0.0245 & 0.0226 & 0.0243 & 0.0228 & 0.0244 & 0.0233 \\
\hline
\end{tabular}

\section{Conclusion}

In this paper, the capacitive sensor was applied to the measurement of rainfall and the sensor's working principle was introduced. Due to the influence of parasitic capacitance and edge effect, the nonlinear error of the sensor's output characteristic curve is large. The first order linear fitting based on the least square method is difficult to describe the input-output characteristics of the sensor accurately. Therefore, BP neural network is used to fit the sensor's characteristic curve. The results show that the BP network has stronger nonlinear mapping ability, and its fitting accuracy is higher than that of the least square method significantly. For capacitive sensors, both the temperature and dielectric substances have a great impact on the sensor's measurement accuracy. In view of these questions, we will design reasonable experiments to do further research.

\section{Acknowledgment}

This work is supported by the National Natural Science Foundation of China (NSFC) (No. 40976062), the Natural Science for Youth Foundation (NSYF) (No. 41406107), and the Natural Science Foundation of Jiangsu Province (No. BK2009062 and No. BK2012513).

\section{Reference}

[1] Cen J S, Wang Q M, Luo H, et al. Preliminary study of raindrop measurement technology and instrument by imaging[J]. Journal of Atmospheric and Environmental Optics, 2011, 06(6):415-422.

[2] Liu X, Wang Y F. Research of automatically piecewise polynomial curve-fitting method based on least-square principle [J]. Science Technology and Engineering, 2014, 14(3):55-58.

[3] Jiang D, Shan Y, Liu X K. Function fitting method for non-linear and temperature compensation 
of force digital sensor [J]. Transducer and Microsystem Technologies, 2016, 35(2):16-18.

[4] Huang K Z, Huang Y. A method based on the inverse function to calibrate a sensor [J]. Sensor World, 2009, 15(5):32-34.

[5] Ding S, Wu Q H, Yang Y L. Realization of digital ECS characteristic curve fitting based on BP neural network [J]. Electronic Design Engineering, 2012, 20(16):64-67.

[6] Jiang Z J, Wang X D. Using BP neural network to realize optic fiber displacement sensor's curve-fitting [J]. Microcomputer and Its Applications, 2012, 31(4):67-70.

[7] Zhang P, Chen M, Qin B. A method to improve static state output characteristic of vibration-type sensor based on BP neural network [J]. Chinese Journal of Sensors and Actuators, 2007, 20(10):2213-2217.

[8] Bao J, Zhao J Y, Zhou H Y. Study on method of curve simulation based on BP network [J]. Computer Engineering and Design, 2005, 26(7):1840-1841. 